\title{
Approximate Controllability of Fractional Sobolev-Type Evolution Equations in Banach Spaces
}

\author{
N. I. Mahmudov \\ Eastern Mediterranean University, Gazimagusa, T.R. North Cyprus, Mersin 10, Turkey \\ Correspondence should be addressed to N. I. Mahmudov; nazim.mahmudov@emu.edu.tr \\ Received 3 January 2013; Accepted 1 February 2013 \\ Academic Editor: Jen-Chih Yao \\ Copyright (C) 2013 N. I. Mahmudov. This is an open access article distributed under the Creative Commons Attribution License, \\ which permits unrestricted use, distribution, and reproduction in any medium, provided the original work is properly cited.

\begin{abstract}
We discuss the approximate controllability of semilinear fractional Sobolev-type differential system under the assumption that the corresponding linear system is approximately controllable. Using Schauder fixed point theorem, fractional calculus and methods of controllability theory, a new set of sufficient conditions for approximate controllability of fractional Sobolev-type differential equations, are formulated and proved. We show that our result has no analogue for the concept of complete controllability. The results of the paper are generalization and continuation of the recent results on this issue.
\end{abstract}

\section{Introduction}

Many social, physical, biological, and engineering problems can be described by fractional partial differential equations. In fact, fractional differential equations are considered as an alternative model to nonlinear differential equations. In the last two decades, fractional differential equations (see Samko et al. [1] and the references therein) have attracted many scientists, and notable contributions have been made to both theory and applications of fractional differential equations.

Recently, the existence of mild solutions and stability and (approximate) controllability of (fractional) semilinear evolution system in Banach spaces have been reported by many researchers; see [2-36]. We refer the reader to ElBorai [3, 4], Balachandran and Park [5], Zhou and Jiao [6, 7] Hernández et al. [8], Wang and Zhou [9], Sakthivel et al. [12, 13], Debbouche and Baleanu [14], Wang et al. [15-21], Kumar and Sukavanam [22], Li and Yong [37], Dauer and Mahmudov [28], Mahmudov [27, 29], and the references therein. Complete controllability of evolution systems of Sobolev type in Banach spaces has been studied by Balachandran and Dauer [23], Ahmed [24], and Feckan et al. [2]. However, the approximate controllability of fractional evolution equations of Sobolev type has not been studied.
Motivated by the above-mentioned papers, we study the approximate controllability of a class of fractional evolution equations of Sobolev type:

$$
\begin{gathered}
{ }^{c} D_{t}^{\alpha}(E x(t))=A x(t)+B u(t)+f(t, x(t)), \quad t \in[0, b], \\
x(0)=x_{0},
\end{gathered}
$$

where $A: D(A) \subset X \rightarrow X$ and $E: D(E) \subset X \rightarrow$ $X$ are linear operators from a Banach space $X$ to $X$. The control function $u$ takes values in a Hilbert space $U$ and $u \in$ $L^{2}([0, b], U) . B: U \rightarrow X$ is a linear bounded operator. The function $f \in C([0, b] \times X, X)$ will be specified in the sequel. The fractional derivative ${ }^{c} D_{t}^{\alpha}, 0<\alpha<1$, is understood in the Caputo sense.

Our aim in this paper is to provide a sufficient condition for the approximate controllability for a class of fractional evolution equations of Sobolev type. It is assumed that $E^{-1}$ is compact, and, consequently, the associated linear control system (35) is not exactly controllable. Therefore, our approximate controllability results have no analogue for the concept of complete controllability. In Section 5, we give an 
example of the system which is not completely controllable, but approximately controllable.

\section{Preliminaries}

Throughout this paper, unless otherwise specified, the following notations will be used. Let $X$ be a separable reflexive Banach space and let $X^{*}$ stand for its dual space with respect to the continuous pairing $\langle\cdot, \cdot\rangle$. We may assume, without loss of generality, that $X$ and $X^{*}$ are smooth and strictly convex, by virtue of a renorming theorem (see, e.g., [37, 38]). In particular, this implies that the duality mapping $J$ of $X$ into $X^{*}$ given by the relations

$$
\|J(z)\|_{*}=\|z\|, \quad\langle J(z), z\rangle=\|z\|^{2}, \quad \forall z \in X
$$

is bijective, homogeneous, demicontinuous, that is, continuous from $X$ with a strong topology, into $X^{*}$ with weak topology, and strictly monotonic. Moreover, $J^{-1}: X^{*} \rightarrow X$ is also duality mapping.

The operators $A: D(A) \subset X \rightarrow X$ and $E: D(E) \subset$ $X \rightarrow X$ satisfy the following hypotheses:

(S1) $A$ and $E$ are linear operators, and $A$ is closed;

(S2) $D(E) \subset D(A)$ and $E$ is bijective;

$(\mathrm{S} 3) E^{-1}: X \rightarrow D(E)$ is compact.

The hypotheses (S1)-(S3) and the closed graph theorem imply the boundedness of the linear operator $A E^{-1}: X \rightarrow$ $X$. Consequently, $-A E^{-1}$ generates a semigroup $\{S(t) ; t \geq 0\}$ in $X$. Assume that $\max _{0 \leq t \leq b}\|S(t)\|=: M$.

Let us recall the following known definitions in fractional calculus. For more details, see [1].

Definition 1. The fractional integral of order $\alpha>0$ with the lower limit 0 for a function $f$ is defined as

$$
I^{\alpha} f(t)=\frac{1}{\Gamma(\alpha)} \int_{0}^{t} \frac{f(s)}{(t-s)^{1-\alpha}} d s, \quad t>0, \alpha>0
$$

provided the right-hand side is pointwise defined on $[0, \infty)$, where $\Gamma$ is the gamma function.

Definition 2. The Caputo derivative of order $\alpha$ for a function $f$ can be written as

$$
\begin{aligned}
{ }^{c} D^{\alpha} f(t) & =\frac{1}{\Gamma(n-\alpha)} \int_{0}^{t} \frac{f^{(n)}(s)}{(t-s)^{1+\alpha-n}} d s \\
& =I^{n-\alpha} f^{(n)}(t), \quad t>0, n-1 \leq \alpha<n .
\end{aligned}
$$

If $f$ is an abstract function with values in $X$, then integrals which appear in the above definitions are taken in Bochner's sense.
For $x \in X$ and $0<\alpha<1$, we define two families $\left\{\mathcal{S}_{E}(t)\right.$ : $t \geq 0\}$ and $\left\{\mathscr{T}_{E}(t): t \geq 0\right\}$ of operators by

$$
\begin{gathered}
\mathcal{S}_{\alpha}(t)=\int_{0}^{\infty} \Psi_{\alpha}(\theta) S\left(t^{\alpha} \theta\right) d \theta, \mathscr{T}_{\alpha}(t) \\
=\alpha \int_{0}^{\infty} \theta \Psi_{\alpha}(\theta) S\left(t^{\alpha} \theta\right) d \theta, \\
\mathcal{S}_{E}(t)=E^{-1} \mathcal{S}_{\alpha}(t), \quad \mathscr{T}_{E}(t)=E^{-1} \mathscr{T}_{\alpha}(t),
\end{gathered}
$$

where

$$
\Psi_{\alpha}(\theta)=\frac{1}{\pi \alpha} \sum_{n=1}^{\infty}(-1)^{n-1} \frac{\Gamma(n \alpha+1)}{n !} \sin (n \pi \alpha), \quad \theta \in(0, \infty),
$$

is the function of Wright type defined on $(0, \infty)$, which satisfies

$$
\begin{gathered}
\Psi_{\alpha}(\theta) \geq 0, \quad \int_{0}^{\infty} \Psi_{\alpha}(\theta) d \theta=1, \\
\int_{0}^{\infty} \theta^{\zeta} \Psi_{\alpha}(\theta) d \theta=\frac{\Gamma(1+\zeta)}{\Gamma(1+\alpha \zeta)}, \quad \zeta \in(-1, \infty) .
\end{gathered}
$$

Lemma 3 (see [2]). The operators $\mathcal{S}_{E}$ and $\mathscr{T}_{E}$ have the following properties.

(i) For any fixed $t \geq 0, S_{E}(t)$ and $T_{E}(t)$ are linear and bounded operators, and

$$
\begin{aligned}
& \left\|\mathcal{S}_{E}(t) x\right\| \leq M\left\|E^{-1}\right\|\|x\|, \\
& \left\|\mathscr{T}_{E}(t) x\right\| \leq \frac{M\left\|E^{-1}\right\|}{\Gamma(\alpha)}\|x\| .
\end{aligned}
$$

(ii) $\left\{\mathcal{S}_{E}(t): t \geq 0\right\}$ and $\left\{\mathscr{T}_{E}(t): t \geq 0\right\}$ are compact.

In this paper, we adopt the following definition of mild solution of (1).

Definition 4. A solution $x(\cdot ; u) \in C([0, b], X)$ is said to be a mild solution of (1) if for any $u \in L^{2}([0, b], U)$, the integral equation

$$
\begin{aligned}
x(t)= & \mathcal{S}_{E}(t) E x_{0} \\
& +\int_{0}^{t}(t-s)^{\alpha-1} \mathscr{T}_{E}(t-s)[B u(s)+f(s, x(s))] d s
\end{aligned}
$$

is satisfied.

Let $x(b ; u)$ be the state value of $(9)$ at terminal time $b$ corresponding to the control $u$. Introduce the set $\mathfrak{R}(b)=$ $\left\{x(b ; u): u \in L_{2}([0, b], U)\right\}$, which is called the reachable set of system (9) at terminal time $b$; its closure in $X$ is denoted by $\overline{\mathfrak{R}(b)}$.

Definition 5. System (1) is said to be approximately controllable on $[0, b]$ if $\overline{\mathfrak{R}(b)}=X$; that is, given an arbitrary $\varepsilon>0$, it is possible to steer from the point $x_{0}$ to within a distance $\varepsilon$ from all points in the state space $X$ at time $b$. 
To investigate the approximate controllability of system (9), we assume the following conditions.

(H4) The function $f:[0, b] \times X \rightarrow X$ satisfies the following:

(a) $f(t, \cdot): X \rightarrow X$ is continuous for each $t \in[0, b]$ and for each $x \in X, f(\cdot, x):[0, b] \rightarrow X$ is strongly measurable;

(b) there is a positive integrable function $n \in$ $L^{1}([0, b],[0,+\infty))$ and a continuous nondecreasing function $\Lambda_{f}:[0, \infty) \rightarrow(0, \infty)$ such that for every $(t, x) \in[0, b] \times X$, we have

$$
\begin{gathered}
\|f(t, x)\| \leq n(t) \Lambda_{f}(\|x\|), \\
\lim \inf _{r \rightarrow \infty} \frac{\Lambda_{f}(r)}{r}=\sigma_{f}<\infty .
\end{gathered}
$$

(H5) The following relationship holds:

$$
\begin{aligned}
\left(1+\frac{1}{\varepsilon} M_{B}^{2} M_{\mathscr{T}}^{2}\right. & \left.\frac{b^{2 \alpha-1}}{2 \alpha-1}\right) \frac{M\left\|E^{-1}\right\|}{\Gamma(\alpha)} \frac{b^{\alpha}}{\alpha} \\
& \times \sigma_{f} \sup _{s \in[0, b]} n(s)<1,
\end{aligned}
$$

here $M_{B}:=\|B\|, M_{\mathscr{T}}:=\left\|\mathscr{T}_{E}\right\|$.

(H6) For every $h \in X, z_{\alpha}(h)=\varepsilon\left(\varepsilon I+\Gamma_{0}^{b} J\right)^{-1}(h)$ converges to zero as $\varepsilon \rightarrow 0^{+}$in strong topology, where

$$
\Gamma_{0}^{b}:=\int_{0}^{b}(b-s)^{2(\alpha-1)} \mathscr{T}_{E}(b-s) B B^{*} \mathscr{T}_{E}^{*}(b-s) d s,
$$

and $z_{\varepsilon}(h)$ is a solution of

$$
\varepsilon z_{\varepsilon}+\Gamma_{0}^{b} J\left(z_{\varepsilon}\right)=\varepsilon h .
$$

\section{Existence Theorem}

In order to formulate the controllability problem in the form suitable for application of fixed point theorem, it is assumed that the corresponding linear system is approximately controllable. Then it will be shown that system (1) is approximately controllable if for all $\varepsilon>0$, there exists a continuous function $x \in C([0, b], X)$ such that

$$
\begin{aligned}
u_{\varepsilon}(t, x)= & (b-t)^{\alpha-1} B^{*} \mathscr{T}_{E}^{*}(b-t) J\left(\left(\varepsilon I+\Gamma_{0}^{b} J\right)^{-1} p(x)\right), \\
x(t)= & \mathcal{S}_{E}(t) E x_{0} \\
& +\int_{0}^{t}(t-s)^{\alpha-1} \mathscr{T}_{E}(t-s)[B u(s)+f(s, x(s))] d s,
\end{aligned}
$$

where

$$
\begin{aligned}
p(x)=h & -\mathcal{S}_{E}(b) E x_{0} \\
& -\int_{0}^{b}(b-s)^{\alpha-1} \mathscr{T}_{E}(b-s) f(s, x(s)) d s .
\end{aligned}
$$

Having noticed this fact, our goal, in this section, is to find conditions for the solvability of (14). It will be shown that the control in (14) drives system (1) from $x_{0}$ to

$$
h-\varepsilon J\left(\left(\varepsilon I+\Gamma_{0}^{b} J\right)^{-1} p(x)\right)
$$

provided that system (14) has a solution.

Theorem 6. Assume that assumptions (S1)-(S3), (H4), (H5) hold and $1 / 2<\alpha \leq 1$. Then there exists a solution to (14).

Proof. The proof of Theorem 6 follows from Lemmas 7-9, infinite dimensional analogue of Arzela-Ascoli theorem, and the Schauder fixed point theorem.

For all $\varepsilon>0$, consider the operator $\Phi_{\varepsilon}: C([0, b], X) \rightarrow$ $C([0, b], X)$ defined as follows:

$$
\begin{aligned}
& \left(\Phi_{\varepsilon} x\right)(t) \\
& :=\mathcal{S}_{E}(t) E x_{0}+\int_{0}^{t}(t-s)^{\alpha-1} \mathscr{T}_{E}(t-s) \\
& \times\left[B u_{\varepsilon}(s, x)+f(s, x(s))\right] d s,
\end{aligned}
$$

where

$$
\begin{gathered}
u_{\varepsilon}(t, x)=(b-t)^{\alpha-1} B^{*} \mathscr{T}_{E}^{*}(b-t) J\left(\left(\varepsilon I+\Gamma_{0}^{b} J\right)^{-1} p(x)\right), \\
p(x)=h-\mathcal{S}_{E}(b) E x_{0} \\
\quad-\int_{0}^{b}(b-s)^{\alpha-1} \mathscr{T}_{E}(b-s) f(s, x(s)) d s .
\end{gathered}
$$

It will be shown that for all $\varepsilon>0$, the operator $\Phi_{\varepsilon}$ : $C([0, b], X) \rightarrow C([0, b], X)$ has a fixed point. To prove this we will employ the Schauder fixed point theorem.

Lemma 7. Under assumptions (S1)-(S3), (H4), (H5), for any $\varepsilon>0$ there exists a positive number $r:=r(\varepsilon)$ such that $\Phi_{\varepsilon}\left(B_{r}\right) \subset B_{r}$.

Proof. Let $\varepsilon>0$ be fixed. If it is not true, then for each $r>0$, there exists a function $z_{r} \in B_{r}$, but $\Phi_{\varepsilon}\left(z_{r}\right) \notin B_{r}$. So for some $t=t(r) \in[0, b]$, one can show that

$$
\begin{aligned}
r \leq & \left\|\left(\Phi_{\varepsilon} z_{r}\right)(t)\right\| \leq\left\|\mathcal{S}_{E}(t) E x_{0}\right\| \\
& +\left\|\int_{0}^{t}(t-s)^{\alpha-1} \mathscr{T}_{E}(t-s) f(s, x(s)) d s\right\| \\
& +\left\|\int_{0}^{t}(t-s)^{\alpha-1} \mathscr{T}_{E}(t-s) B u_{\varepsilon}(s, x) d s\right\| \\
= & : I_{1}+I_{2}+I_{3} .
\end{aligned}
$$


Let us estimate $I_{i}, i=1,2,3$. By the assumption (H4), we have

$$
\begin{gathered}
I_{1} \leq\left\|\mathcal{S}_{E}(t) E x_{0}\right\| \leq M\left\|E^{-1}\right\|\left\|E x_{0}\right\|, \\
I_{2} \leq \int_{0}^{t}\left\|(t-s)^{\alpha-1} \mathscr{T}_{E}(t-s)(t-s) f(s, x(s))\right\| d s \\
\leq \frac{M\left\|E^{-1}\right\|}{\Gamma(\alpha)} \int_{0}^{t}(t-s)^{\alpha-1}\|f(s, x(s))\| d s \\
\leq \frac{M\left\|E^{-1}\right\|}{\Gamma(\alpha)} \int_{0}^{t}(t-s)^{\alpha-1} n(s) \Lambda_{f}(\|x(s)\|) d s \\
\leq \frac{M\left\|E^{-1}\right\| b^{\alpha}}{\Gamma(\alpha)} \frac{b_{f}(r) \sup _{s \in J} n(s) .}{\alpha}
\end{gathered}
$$

Combining the estimates (19)-(21) yields

$$
\begin{aligned}
I_{1}+I_{2}< & M\left\|E^{-1}\right\|\left\|E x_{0}\right\| \\
& +\frac{M\left\|E^{-1}\right\|}{\Gamma(\alpha)} \frac{b^{\alpha}}{\alpha} \Lambda_{f}(r) \sup _{s \in[0, b]} n(s):=\Delta .
\end{aligned}
$$

On the other hand,

$$
\begin{aligned}
I_{3} \leq & \int_{0}^{t}\left\|(t-s)^{\alpha-1} \mathscr{T}_{E}(t-s) B u_{\varepsilon}(s, x)\right\| d s \\
= & \int_{0}^{t} \|(t-s)^{\alpha-1}(b-s)^{\alpha-1} \mathscr{T}_{E}(t-s) \\
& \times B B^{*} \mathscr{T}_{E}^{*}(b-t) J\left(\left(\varepsilon I+\Gamma_{0}^{b} J\right)^{-1} p(x)\right) \| d s \\
\leq & \int_{0}^{t}\left\|(t-s)^{\alpha-1}(b-s)^{\alpha-1} \mathscr{T}_{E}(t-s) B B^{*} \mathscr{T}_{E}^{*}(b-t)\right\| d s \\
\leq & M_{B}^{2} M_{\mathscr{T}}^{2} \frac{b^{2 \alpha-1}}{2 \alpha-1}\left\|J\left(\left(\varepsilon I+\Gamma_{0}^{b} J\right)^{-1} p(x)\right)\right\| \\
= & M_{B}^{2} M_{\mathscr{T}}^{2} \frac{b^{2 \alpha-1}}{2 \alpha-1}\left\|\left(\varepsilon I+\Gamma_{0}^{b} J\right)^{-1} p(x)\right\| \\
\leq & \frac{1}{\varepsilon} M_{B}^{2} M_{\mathscr{T}}^{2} \frac{b^{2 \alpha-1}}{2 \alpha-1}\|p(x)\| \\
\leq & \frac{1}{\varepsilon} M_{B}^{2} M_{\mathscr{T}}^{2} \frac{b^{2 \alpha-1}}{2 \alpha-1} \Delta .
\end{aligned}
$$

Thus,

$$
\begin{aligned}
r & \leq\left\|\left(\Phi_{\varepsilon} z_{r}\right)(t)\right\| \leq \Delta+\frac{1}{\varepsilon} M_{B}^{2} M_{\mathscr{T}}^{2} \frac{b^{2 \alpha-1}}{2 \alpha-1} \Delta \\
& =\left(1+\frac{1}{\varepsilon} M_{B}^{2} M_{\mathscr{T}}^{2} \frac{b^{2 \alpha-1}}{2 \alpha-1}\right) \Delta .
\end{aligned}
$$

Dividing both sides by $r$ and taking $r \rightarrow \infty$, we obtain that

$$
\left(1+\frac{1}{\varepsilon} M_{B}^{2} M_{\mathscr{T}}^{2} \frac{b^{2 \alpha-1}}{2 \alpha-1}\right) \frac{M\left\|E^{-1}\right\|}{\Gamma(\alpha)} \frac{b^{\alpha}}{\alpha} \sigma_{f} \sup _{s \in[0, b]} n(s) \geq 1
$$

which is a contradiction by assumption (H5). Thus, $\Phi_{\varepsilon}\left(B_{r}\right) \subset$ $B_{r}$ for some $r>0$.

Lemma 8. Let assumptions (S1)-(S3), (H4), (H5) hold. Then the set $\left\{\Phi_{\varepsilon} z: z \in B_{r}\right\}$ is an equicontinuous family of functions on $[0, b]$.

Proof. Let $0<\eta<t<b$ and $\delta>0$ such that

$$
\left\|\mathscr{T}_{E}\left(s_{1}\right)-\mathscr{T}_{E}\left(s_{2}\right)\right\|<\eta
$$

for every $s_{1}, s_{2} \in[0, b]$ with $\left|s_{1}-s_{2}\right|<\delta$. For $z \in B_{r}, 0<|h|<$ $\delta, t+h \in[0, b]$, we have

$$
\begin{aligned}
& \left\|\left(\Phi_{\varepsilon} z\right)(t+h)-\left(\Phi_{\varepsilon} z\right)(t)\right\| \\
& \leq \| \int_{0}^{t}\left((t+h-s)^{\alpha-1}-(t-s)^{\alpha-1}\right) \\
& \quad \times \mathscr{T}_{E}(t+h-s)\left[B u_{\varepsilon}(s, z)+f(s, z(s))\right] d s \| \\
& +\| \int_{t}^{t+h}(t+h-s)^{\alpha-1} \mathscr{T}_{E}(t+h-s) \\
& \quad \times\left[B u_{\varepsilon}(s, z)+f(s, z(s))\right] d s \| \\
& +\| \int_{0}^{t}(t-s)^{\alpha-1}\left(\mathscr{T}_{E}(t+h-s)-\mathscr{T}_{E}(t-s)\right) \\
& \quad \times\left[B u_{\varepsilon}(s, z)+f(s, z(s))\right] d s \| .
\end{aligned}
$$

Applying Lemma 3 and the Holder inequality, we obtain

$$
\begin{aligned}
& \left\|\left(\Phi_{\varepsilon} z\right)(t+h)-\left(\Phi_{\varepsilon} z\right)(t)\right\| \\
& \leq \frac{M\left\|E^{-1}\right\|}{\Gamma(\alpha)} \Lambda_{f}(r) \\
& \quad \times \int_{0}^{t}\left((t+h-s)^{\alpha-1}-(t-s)^{\alpha-1}\right) n(s) d s \\
& +\frac{M\left\|E^{-1}\right\|}{\Gamma(\alpha)} \frac{1}{\varepsilon} M_{B} M_{\mathscr{T}} \Delta
\end{aligned}
$$




$$
\begin{aligned}
& \times \int_{0}^{t}\left((t+h-s)^{\alpha-1}-(t-s)^{\alpha-1}\right)(b-s)^{\alpha-1} d s \\
& +\frac{M\left\|E^{-1}\right\|}{\Gamma(\alpha)} \Lambda_{f}(r) \int_{t}^{t+h}(t+h-s)^{\alpha-1} n(s) d s \\
& +\frac{M\left\|E^{-1}\right\|}{\Gamma(\alpha)} \frac{1}{\varepsilon} M_{B} M_{\mathscr{T}} \Delta \\
& \times \int_{t}^{t+h}(t+h-s)^{\alpha-1}(b-s)^{\alpha-1} d s \\
& +\frac{\eta T^{\alpha}}{\alpha} \Lambda_{f}(r) \int_{0}^{t}(t-s)^{\alpha-1} n(s) d s+\frac{\eta T^{\alpha}}{\alpha} \frac{1}{\varepsilon} M_{B} M_{\mathscr{T}} \Delta \\
& \times \int_{0}^{t}(t-s)^{\alpha-1}(b-s)^{\alpha-1} d s .
\end{aligned}
$$

Therefore, for $\varepsilon$ sufficiently small, the right-hand side of (28) tends to zero as $h \rightarrow 0$. On the other hand, the compactness of $\mathscr{T}_{E}(t), t>0$, implies the continuity in the uniform operator topology. Thus, the set $\left\{\Phi_{\varepsilon} z: z \in B_{r}\right\}$ is equicontinuous.

Lemma 9. Let assumptions (S1)-(S3), (H4), (H5) hold. Then $\Phi_{\varepsilon}$ maps $B_{r}$ onto a precompact set in $B_{r}$.

Proof. Let $0<t \leq b$ be fixed and let $\lambda$ be a real number satisfying $0<\lambda<t$. For $\delta>0$, define an operator $\Phi_{\varepsilon}^{\lambda, \delta}$ on $B_{r}$ by

$$
\begin{aligned}
& \left(\Phi_{\varepsilon}^{\lambda, \delta} z\right)(t) \\
& =\alpha \int_{0}^{t-\lambda} E^{-1} \int_{\delta}^{\infty} \theta(t-s)^{\alpha-1} \Psi_{\alpha}(\theta) S\left((t-s)^{\alpha} \theta\right) \\
& \quad \times\left[B u_{\varepsilon}(s, z)+f(s, z(s))\right] d s \\
& =\alpha E^{-1} S\left(\lambda^{\alpha} \delta\right) \\
& \quad \times \int_{0}^{t-\lambda} \int_{\delta}^{\infty} \theta(t-s)^{\alpha-1} \Psi_{\alpha}(\theta) S\left((t-s)^{\alpha} \theta-\lambda^{\alpha} \delta\right) \\
& \quad \times\left[B u_{\varepsilon}(s, z)+f(s, z(s))\right] d s .
\end{aligned}
$$

Since $E^{-1}$ is a compact operator, the set $\left\{\left(\Phi_{\varepsilon}^{\lambda, \delta} z\right)(t): z \in B_{r}\right\}$ is precompact in $X$ for every $0<\lambda<t, \delta>0$. Moreover, for each $z \in B_{r}$, we have

$$
\begin{aligned}
& \|\left(\Phi_{\varepsilon} z\right)(t)-\left(\Phi_{\varepsilon}^{\lambda, \delta} z\right)(t) \| \\
& \leq \alpha \| \int_{0}^{t} \int_{0}^{\delta} \theta(t-s)^{\alpha-1} \Psi_{\alpha}(\theta) S\left((t-s)^{\alpha} \theta\right) \\
& \quad \times\left[B u_{\varepsilon}(s, z)+f(s, z(s))\right] d \theta d s \|
\end{aligned}
$$

$$
\begin{aligned}
& +\alpha \| \int_{t-\lambda}^{t} \int_{\delta}^{\infty} \theta(t-s)^{\alpha-1} \Psi_{\alpha}(\theta) S\left((t-s)^{\alpha} \theta\right) \\
& \quad \times\left[B u_{\varepsilon}(s, z)+f(s, z(s))\right] d \theta d s \| \\
& =: J_{1}+J_{2} .
\end{aligned}
$$

A similar argument, as before, is as follows:

$$
\begin{aligned}
J_{1} \leq \alpha M \int_{0}^{t}(t-s)^{\alpha-1}\left(\left\|B u_{\varepsilon}(s, z)\right\|+\|f(s, z(s))\|\right) d s \\
\quad \times\left(\int_{0}^{\delta} \theta \Psi_{\alpha}(\theta) d \theta\right) \\
\leq \alpha M\left(\frac{1}{\varepsilon} M_{B} M_{\mathscr{T}} \Delta \int_{0}^{t}(t-s)^{\alpha-1}(b-s)^{\alpha-1} d s\right. \\
\left.+\Lambda_{f}(r) \int_{0}^{t}(t-s)^{\alpha-1} n(s) d s\right)\left(\int_{0}^{\delta} \theta \Psi_{\alpha}(\theta) d \theta\right)
\end{aligned}
$$

$$
\begin{gathered}
J_{2} \leq \alpha M \int_{t-\lambda}^{t}(t-s)^{\alpha-1}\left(\left\|B u_{\varepsilon}(s, z)\right\|+\|f(s, z(s))\|\right) d s \\
\times\left(\int_{\delta}^{\infty} \theta \eta_{\alpha}(\theta) d \theta\right) \\
\leq \frac{\alpha M}{\Gamma(1+\alpha)}\left(\frac{1}{\varepsilon} M_{B} M_{\mathscr{T}} \Delta \int_{t-\lambda}^{t}(t-s)^{\alpha-1}(b-s)^{\alpha-1} d s\right. \\
\left.+\Lambda_{f}(r) \int_{t-\lambda}^{t}(t-s)^{\alpha-1} n(s) d s\right),
\end{gathered}
$$

where we have used the equality

$$
\int_{0}^{\infty} \theta_{\alpha}^{\beta} \Psi_{\alpha}(\theta) d \theta=\frac{\Gamma(1+\beta)}{\Gamma(1+\alpha \beta)} .
$$

From (30)-(32), one can see that for each $z \in B_{r}$,

$$
\left\|\left(\Phi_{\varepsilon} z\right)(t)-\left(\Phi_{\varepsilon}^{\lambda, \delta} z\right)(t)\right\| \longrightarrow 0 \quad \text { as } \lambda \longrightarrow 0^{+}, \delta \longrightarrow 0^{+}
$$

Therefore, there are relatively compact sets arbitrary close to the set $\left\{\left(\Phi_{\varepsilon} z\right)(t): z \in B_{r}\right\}$; hence, the set $\left\{\left(\Phi_{\varepsilon} z\right)(t): z \in B_{r}\right\}$ is also precompact in $X$.

\section{Main Results}

Consider the following linear fractional differential system:

$$
\begin{gathered}
D_{t}^{\alpha} E x(t)=A x(t)+B u(t), \quad t \in(0, b], \\
x(0)=x_{0} .
\end{gathered}
$$


It is convenient at this point to introduce the controllability and resolvent operators associated with (35) as

$$
\begin{aligned}
& L_{0}^{b}=\int_{0}^{b}(b-s)^{\alpha-1} \mathscr{T}_{E}(b-s) B u(s) d s: L^{2}([0, b], U) \longrightarrow X, \\
& \Gamma_{0}^{b}=L_{0}^{b}\left(L_{0}^{b}\right)^{*}=\int_{0}^{b}(b-s)^{2(\alpha-1)} \mathscr{T}_{E}(b-s) \\
& \times B B^{*} \mathscr{T}_{E}^{*}(b-s) d s: X \longrightarrow X,
\end{aligned}
$$

respectively, where $B^{*}$ denotes the adjoint of $B$ and $\mathscr{T}_{\alpha}^{*}(t)$ is the adjoint of $\mathscr{T}_{\alpha}(t)$. It is straightforward that the operator $L_{0}^{b}$ is a linear bounded operator for $1 / 2<\alpha \leq 1$.

Theorem 10 (see [27]). The following three conditions are equivalent.

(i) $\Gamma$ is positive; that is, $\left\langle z^{*}, \Gamma z^{*}\right\rangle>0$ for all nonzero $z^{*} \epsilon$ $X^{*}$.

(ii) For all $h \in X, J\left(z_{\varepsilon}(h)\right)$ converges to zero as $\varepsilon \rightarrow 0^{+}$in the weak topology, where $z_{\varepsilon}(h)=\varepsilon(\varepsilon I+\Gamma J)^{-1}(h)$ is a solution of (13).

(iii) For all $h \in X, z_{\varepsilon}(h)=\varepsilon(\varepsilon I+\Gamma J)^{-1}(h)$ converges to zero as $\varepsilon \rightarrow 0^{+}$in the strong topology.

Remark 11. It is known that Theorem 10 (i) holds if and only if $\overline{\operatorname{Im} L_{0}^{b}}=X$. In other words, Theorem 10 (i) holds if and only if the corresponding linear system is approximately controllable on $[0, b]$. Consequently, assumption (H6) is equivalent to the approximate controllability of the linear system (35).

Theorem 12 (see [27]). Let $p: X \rightarrow X$ be a nonlinear operator. Assume that $z_{\varepsilon}$ is a solution of the following equation:

$$
\begin{gathered}
\varepsilon z_{\varepsilon}+\Gamma_{0}^{T} J\left(z_{\varepsilon}\right)=\varepsilon p\left(z_{\varepsilon}\right), \\
\left\|p\left(z_{\varepsilon}\right)-q\right\| \longrightarrow 0 \quad \text { as } \varepsilon \longrightarrow 0^{+}, q \in X .
\end{gathered}
$$

Then there exists a subsequence of the sequence $\left\{z_{\varepsilon}\right\}$ strongly converging to zero as $\varepsilon \rightarrow 0^{+}$.

We are now in a position to state and prove the main result of the paper.

Theorem 13. Let $1 / 2<\alpha \leq 1$. Suppose that conditions (S1)(S3), (H4)-(H5) are satisfied. Besides, assume additionally that there exists $N \in L^{\infty}([0, b],[0,+\infty))$ such that

$$
\sup _{x \in X}\|f(t, x)\| \leq N(t), \quad \text { for a.e. } t \in[0, b] .
$$

Then system (1) is approximately controllable on $[0, b]$.
Proof. Let $x^{\varepsilon}$ be a fixed point of $\Phi_{\varepsilon}$ in $B_{r(\varepsilon)}$. Then $x^{\varepsilon}$ is a mild solution of $(1)$ on $[0, b]$ under the control

$$
\begin{gathered}
u_{\varepsilon}\left(t, x^{\varepsilon}\right)=(b-t)^{\alpha-1} B^{*} \mathscr{T}_{E}^{*}(b-t) J\left(\left(\varepsilon I+\Gamma_{0}^{b} J\right)^{-1} p\left(x^{\varepsilon}\right)\right), \\
p\left(x^{\varepsilon}\right)=h-\mathcal{S}_{E}(b) x_{0} \\
\quad-\int_{0}^{b}(b-s)^{\alpha-1} \mathscr{T}_{E}(b-s) f\left(s, x^{\varepsilon}(s)\right) d s
\end{gathered}
$$

and satisfies the following equality:

$$
\begin{aligned}
x^{\varepsilon}(b)= & \mathcal{S}_{E}(b) x_{0}+\int_{0}^{b}(b-s)^{\alpha-1} \mathscr{T}_{E}(b-s) \\
& \times\left[B u_{\varepsilon}\left(s, x^{\varepsilon}\right)+f\left(s, x^{\varepsilon}(s)\right)\right] d s \\
= & \mathcal{S}_{E}(b) x_{0}+\left(-\varepsilon I+\varepsilon I+\Gamma_{0}^{b} J\right)\left(\left(\varepsilon I+\Gamma_{0}^{b} J\right)^{-1} p\left(x^{\varepsilon}\right)\right) \\
& +\int_{0}^{b}(b-s)^{\alpha-1} \mathscr{T}_{E}(b-s) f\left(s, x^{\varepsilon}(s)\right) d s \\
= & h-\varepsilon\left(\varepsilon I+\Gamma_{0}^{b} J\right)^{-1} p\left(x^{\varepsilon}\right) .
\end{aligned}
$$

In other words, $z_{\varepsilon}=h-x^{\varepsilon}(b)$ is a solution of

$$
\left(\varepsilon I+\Gamma_{0}^{b} J\right)\left(z_{\varepsilon}\right)=\varepsilon p\left(x^{\varepsilon}\right) .
$$

By our assumption,

$$
\int_{0}^{b}\left\|f\left(s, x^{\varepsilon}(s)\right)\right\|^{2} d s \leq \int_{0}^{T} N^{2}(s) d s .
$$

Consequently, the sequence $\left\{f\left(\cdot, x^{\varepsilon}(\cdot)\right)\right\}$ is bounded. Then there is a subsequence still denoted by $\left\{f\left(\cdot, x^{\varepsilon}(\cdot)\right)\right\}$ and weakly converges to, say, $f(\cdot)$ in $L^{2}([0, b], X)$. Then

$$
\begin{aligned}
& \left\|p\left(x^{\varepsilon}\right)-q\right\| \\
& =\left\|\int_{0}^{b}(b-s)^{\alpha-1} \mathscr{T}_{E}(b-s)\left[f\left(s, x^{\varepsilon}(s)\right)-f(s)\right] d s\right\| \\
& \leq \sup _{0 \leq t \leq b} \| \int_{0}^{t}(t-s)^{\alpha-1} \mathscr{T}_{E}(t-s) \\
& \quad \times\left[f\left(s, x^{\varepsilon}(s)\right)-f(s)\right] d s \| \longrightarrow 0,
\end{aligned}
$$

where

$$
q=h-\mathcal{S}_{E} E x_{0}-\int_{0}^{b}(b-s)^{\alpha-1} \mathscr{T}_{E}(b-s) f(s) d s
$$

as $\varepsilon \rightarrow 0^{+}$because of the compactness of an operator $f(\cdot) \rightarrow$ $\int_{0}^{\cdot}(\cdot-s)^{\alpha-1} \mathscr{T}_{E}(\cdot-s) f(s) d s: L_{2}([0, b], X) \rightarrow C([0, b], X)$. Then by Theorem 12 for any $h \in X$,

$$
\left\|x^{\varepsilon}(b)-h\right\|=\left\|z_{\varepsilon}\right\| \longrightarrow 0
$$

as $\varepsilon \rightarrow 0^{+}$. This gives the approximate controllability. The theorem is proved. 
Remark 14. Theorem 13 assumes that the operator $E^{-1}$ is compact and, consequently, the associated linear control system (35) is not exactly controllable. Therefore, Theorem 13 has no analogue for the concept of exact controllability.

Remark 15. In order to describe various real-world problems in physical and engineering sciences subject to abrupt changes at certain instants during the evolution process, fractional impulsive differential equations have been used for the system model. Our result can be extended to study the complete and approximate controllability of nonlinear fractional impulsive differential equations of Sobolev type; see $[35,36]$.

\section{Applications}

Example 16. Let $X=U=L^{2}[0, \pi]$. Consider the following fractional partial differential equation with control:

$$
\begin{array}{r}
{ }^{c} D_{t}^{3 / 4}\left(x(t, \theta)-x_{\theta \theta}(t, \theta)\right) \\
=x_{\theta \theta}(t, \theta)+g(t, x(t, \theta))+u(t, \theta), \\
x(t, 0)=x(t, \pi)=0, \\
x(0, \theta)=\phi(\theta),: 0 \leq t \leq b,: 0 \leq \theta \leq \pi .
\end{array}
$$

Define $A: D(A) \subset X \rightarrow X$ by $A:=x_{\theta \theta}$ and $E:$ $D(E) \subset X \rightarrow X$ by $E x:=x-x_{\theta \theta}$, where each domain, $D(A)$ and $D(E)$, is given by

$\left\{x \in X: x, x_{\theta}\right.$ are absolutely continuous,

$$
\left.x_{\theta \theta} \in X, x(t, 0)=x(t, \pi)=0\right\} .
$$

$A$ and $E$ can be written as follows:

$$
\begin{gathered}
A x:=\sum_{n=1}^{\infty}-n^{2}\left\langle x, e_{n}\right\rangle e_{n}, \quad x \in D(A), \\
E x=\sum_{n=1}^{\infty}\left(1+n^{2}\right)\left\langle x, e_{n}\right\rangle e_{n}, \quad x \in D(E),
\end{gathered}
$$

respectively, where $e_{n}(\theta):=\sqrt{2 / \pi} \sin n \theta, n=1,2, \ldots$, is the orthonormal set of eigenvalues of $A$. Moreover, for any $x \in X$, we have

$$
\begin{aligned}
E^{-1} x & =\sum_{n=1}^{\infty} \frac{1}{1+n^{2}}\left\langle x, e_{n}\right\rangle e_{n}, \\
A E^{-1} x & =\sum_{n=1}^{\infty} \frac{-n^{2}}{1+n^{2}}\left\langle x, e_{n}\right\rangle e_{n}, \\
S(t) x & =\sum_{n=1}^{\infty} \exp \left(\frac{-n^{2}}{1+n^{2}} t\right)\left\langle x, e_{n}\right\rangle e_{n}, \\
\mathscr{T}_{E}(t) & =\frac{3}{4} \int_{0}^{\infty} E^{-1} \theta \xi_{3 / 4}(\theta) S\left(t^{3 / 4} \theta\right) d \theta, \\
\mathscr{T}_{E}(t) x & =\frac{3}{4} \sum_{n=1}^{\infty} \frac{1}{1+n^{2}}
\end{aligned}
$$

$$
\begin{aligned}
& \times \int_{0}^{\infty} \theta \xi_{3 / 4}(\theta) \exp \left(\frac{-n^{2}}{1+n^{2}} t^{3 / 4} \theta\right) d \theta \\
& \times\left\langle x, e_{n}\right\rangle e_{n} \\
= & -\sum_{n=1}^{\infty} \frac{1}{n^{2}} t^{1 / 4} \\
& \times \int_{0}^{\infty} \xi_{3 / 4}(\theta) \frac{d}{d t} \exp \left(\frac{-n^{2}}{1+n^{2}} t^{3 / 4} \theta\right) d \theta \\
& \times\left\langle x, e_{n}\right\rangle e_{n}, \\
\Gamma_{0}^{b} & =\int_{0}^{b}(b-s)^{2(\alpha-1)} \mathscr{T}(b-s) \mathscr{T}(b-s) d s \\
= & \int_{0}^{b} s^{2(\alpha-1)} \mathscr{T}(s) \mathscr{T}(s) d s \\
= & \int_{0}^{b} s^{2(\alpha-1)} \frac{3}{4} \int_{0}^{\infty} E^{-1} \theta \xi_{3 / 4}(\theta) S\left(s^{3 / 4} \theta\right) d \theta \\
& \times \frac{3}{4} \int_{0}^{\infty} E^{-1} \theta \xi_{3 / 4}(\theta) S\left(s^{3 / 4} \theta\right) d \theta d s .
\end{aligned}
$$

It is clear that $E^{-1}$ is compact. The linear system corresponding to (47) is completely controllable if and only if there exists $\gamma>0$ such that $\left\langle\Gamma_{0}^{b} x, x\right\rangle \geq \gamma\|x\|^{2}$ for all $x \in X$. Assume

$$
\begin{aligned}
& \left\langle\Gamma_{0}^{b} x, x\right\rangle \\
& =\frac{9}{16} \sum_{n=1}^{\infty} \frac{1}{\left(1+n^{2}\right)^{2}} \\
& \quad \times \int_{0}^{b} s^{2(\alpha-1)}\left(\int_{0}^{\infty} \theta \xi_{3 / 4}(\theta) \exp \left(\frac{-n^{2}}{1+n^{2}} s^{3 / 4} \theta\right) d \theta\right)^{2} d s \\
& \quad \times\left\langle x, e_{n}\right\rangle^{2} \\
& \geqslant \\
& \gamma \sum_{n=1}^{\infty}\left\langle x, e_{n}\right\rangle^{2} .
\end{aligned}
$$

Then

$$
\begin{aligned}
& \frac{9}{16} \frac{1}{\left(1+n^{2}\right)^{2}} \\
& \quad \times \int_{0}^{b} s^{2(\alpha-1)}\left(\int_{0}^{\infty} \theta \xi_{3 / 4}(\theta) \exp \left(\frac{-n^{2}}{1+n^{2}} s^{3 / 4} \theta\right) d \theta\right)^{2} d s \\
& \quad \geqslant \gamma, \\
& \quad \times \frac{9}{16} \frac{1}{\left(1+n^{2}\right)^{2}} \int_{0}^{b} s^{-1 / 2}\left(\int_{0}^{\infty} \theta \xi_{3 / 4}(\theta) \exp \left(\frac{-n^{2}}{1+n^{2}} s^{3 / 4} \theta\right) d \theta\right)^{2} d s
\end{aligned}
$$




$$
\begin{aligned}
& \leq \frac{9}{16} \frac{1}{\left(1+n^{2}\right)^{2}} \\
& \quad \times \int_{0}^{b} s^{-1 / 2}\left(\int_{0}^{\infty} \theta \xi_{3 / 4}(\theta) d \theta\right)^{2} d s \longrightarrow 0, \\
& \quad \text { as } n \longrightarrow \infty, \Longrightarrow \gamma=0 \text { (contradiction), }
\end{aligned}
$$

and no such $\gamma>0$ exists which satisfies (51), and hence the linear system corresponding to (47) is never completely controllable. We show that the associated linear system is approximately controllable on $[0, b]$. We need to show that $(b-s)^{\alpha-1} B^{*} \mathscr{T}_{E}^{*}(b-s) x=0,0 \leq s<b \Rightarrow x=0$. Indeed,

$$
\begin{aligned}
&(b-s)^{\alpha-1} B^{*} \mathscr{T}_{E}^{*}(b-s) x \\
&=(b-s)^{\alpha-1} \frac{3}{4} \int_{0}^{\infty} \theta \xi_{3 / 4}(\theta) \sum_{n=1}^{\infty} \frac{1}{1+n^{2}} \exp \left(\frac{-n^{2}}{1+n^{2}} s^{3 / 4} \theta\right) \\
& \quad \times\left\langle x, e_{n}\right\rangle e_{n}=0, \\
& \frac{3}{4} \int_{0}^{\infty} \theta \xi_{3 / 4}(\theta) \sum_{n=1}^{\infty} \frac{1}{1+n^{2}} \exp \left(\frac{-n^{2}}{1+n^{2}} s^{3 / 4} \theta\right)\left\langle x, e_{n}\right\rangle e_{n}=0, \\
&\left\langle x, e_{n}\right\rangle=0 \Longrightarrow x=0 .
\end{aligned}
$$

Next, we suppose

(H6) $g:[0, b] \times R \rightarrow R$. For each $x \in R, g(\cdot, x)$ is measurable and for each $t \in[0, b], g(t, \cdot)$ is continuous. Moreover, $\sup _{x \in R}\|g(t, x)\| \leq N(t)$, for a.e. $t \in[0, b]$.

Define $f:[0, b] \times X \rightarrow X$ by $f(t, x)(\theta)=g(t, x(t, \theta))$. Now, system (47) can be written in the abstract form (1). Clearly, all the assumptions in Theorem 13 are satisfied if (H6) holds. Then system (47) is approximately controllable on $[0, b]$.

\section{References}

[1] S. G. Samko, A. A. Kilbas, and O. I. Marichev, Fractional Integrals and Derivatives, Gordon and Breach Science Publishers, London, UK, 1993.

[2] M. Feckan, J. R. Wang, and Y. Zhou, "Controllability of fractional functional evolution equations of sobolev type via characteristic solution operators," Journal of Optimization Theory and Applications, 2012.

[3] M. M. El-Borai, "Some probability densities and fundamental solutions of fractional evolution equations," Chaos, Solitons and Fractals, vol. 14, no. 3, pp. 433-440, 2002.

[4] M. M. El-Borai, "The fundamental solutions for fractional evolution equations of parabolic type," Journal of Applied Mathematics and Stochastic Analysis, no. 3, pp. 197-211, 2004.

[5] K. Balachandran and J. Y. Park, "Controllability of fractional integrodifferential systems in Banach spaces," Nonlinear Analysis: Hybrid Systems, vol. 3, no. 4, pp. 363-367, 2009.

[6] Y. Zhou and F. Jiao, "Existence of mild solutions for fractional neutral evolution equations," Computers \& Mathematics with Applications, vol. 59, no. 3, pp. 1063-1077, 2010.
[7] Y. Zhou and F. Jiao, "Nonlocal Cauchy problem for fractional evolution equations," Nonlinear Analysis: Real World Applications, vol. 11, no. 5, pp. 4465-4475, 2010.

[8] E. Hernández, D. O’Regan, and K. Balachandran, “On recent developments in the theory of abstract differential equations with fractional derivatives," Nonlinear Analysis: Theory, Methods \& Applications, vol. 73, no. 10, pp. 3462-3471, 2010.

[9] J. Wang and Y. Zhou, "A class of fractional evolution equations and optimal controls," Nonlinear Analysis: Real World Applications, vol. 12, no. 1, pp. 262-272, 2011.

[10] J. Wang and Y. Zhou, "Existence and controllability results for fractional semilinear differential inclusions," Nonlinear Analysis: Real World Applications, vol. 12, no. 6, pp. 3642-3653, 2011.

[11] J. Wang and Y. Zhou, "Analysis of nonlinear fractional control systems in Banach spaces," Nonlinear Analysis: Theory, Methods \& Applications, vol. 74, no. 17, pp. 5929-5942, 2011.

[12] R. Sakthivel, Y. Ren, and N. I. Mahmudov, "On the approximate controllability of semilinear fractional differential systems," Computers \& Mathematics with Applications, vol. 62, no. 3, pp. 1451-1459, 2011.

[13] R. Sakthivel, N. I. Mahmudov, and J. J. Nieto, "Controllability for a class of fractional-order neutral evolution control systems," Applied Mathematics and Computation, vol. 218, no. 20, pp. 10334-10340, 2012.

[14] A. Debbouche and D. Baleanu, "Controllability of fractional evolution nonlocal impulsive quasilinear delay integrodifferential systems," Computers \& Mathematics with Applications, vol. 62, no. 3, pp. 1442-1450, 2011.

[15] J. Wang, Y. Zhou, and M. Medved, "On the solvability and optimal controls of fractional integrodifferential evolution systems with infinite delay," Journal of Optimization Theory and Applications, vol. 152, no. 1, pp. 31-50, 2012.

[16] J. Wang and Y. Zhou, "Mittag-Leffler-Ulam stabilities of fractional evolution equations," Applied Mathematics Letters, vol. 25, no. 4, pp. 723-728, 2012.

[17] J. Wang, Y. Zhou, and W. Wei, "Optimal feedback control for semilinear fractional evolution equations in Banach spaces," Systems \& Control Letters, vol. 61, no. 4, pp. 472-476, 2012.

[18] J. Wang, Z. Fan, and Y. Zhou, "Nonlocal controllability of semilinear dynamic systems with fractional derivative in Banach spaces," Journal of Optimization Theory and Applications, vol. 154, no. 1, pp. 292-302, 2012.

[19] J. Wang and Y. Zhou, "Complete controllability of fractional evolution systems," Communications in Nonlinear Science and Numerical Simulation, vol. 17, pp. 4346-4355, 2012.

[20] J. Wang, Y. Zhou, and W. Wei, "Fractional Schrödinger equations with potential and optimal controls," Nonlinear Analysis: Real World Applications, vol. 13, no. 6, pp. 2755-2766, 2012.

[21] R.-N. Wang, D.-H. Chen, and T.-J. Xiao, "Abstract fractional Cauchy problems with almost sectorial operators," Journal of Differential Equations, vol. 252, no. 1, pp. 202-235, 2012.

[22] S. Kumar and N. Sukavanam, "Approximate controllability of fractional order semilinear systems with bounded delay," Journal of Differential Equations, vol. 252, no. 11, pp. 6163-6174, 2012.

[23] K. Balachandran and J. P. Dauer, "Controllability of functionaldifferential systems of Sobolev type in Banach spaces," Kybernetika, vol. 34, no. 3, pp. 349-357, 1998.

[24] H. Ahmed, "Controllability for Sobolev type fractional integrodifferential systems in a Banach space," Advances in Difference Equations, vol. 2012, article 167, 2012. 
[25] A. E. Bashirov and N. I. Mahmudov, "On concepts of controllability for deterministic and stochastic systems," SIAM Journal on Control and Optimization, vol. 37, no. 6, pp. 1808-1821, 1999.

[26] A. E. Bashirov, N. Mahmudov, N. Şemi, and H. Etikan, "Partial controllability concepts," International Journal of Control, vol. 80, no. 1, pp. 1-7, 2007.

[27] N. I. Mahmudov, "Approximate controllability of semilinear deterministic and stochastic evolution equations in abstract spaces," SIAM Journal on Control and Optimization, vol. 42, no. 5, pp. 1604-1622, 2003.

[28] J. P. Dauer and N. I. Mahmudov, "Approximate controllability of semilinear functional equations in Hilbert spaces," Journal of Mathematical Analysis and Applications, vol. 273, no. 2, pp. 310327, 2002.

[29] N. I. Mahmudov, "Approximate controllability of evolution systems with nonlocal conditions," Nonlinear Analysis: Theory, Methods \& Applications, vol. 68, no. 3, pp. 536-546, 2008.

[30] R. Sakthivel, N. I. Mahmudov, and J. H. Kim, "On controllability of second order nonlinear impulsive differential systems," Nonlinear Analysis: Theory, Methods \& Applications, vol. 71, no. 1-2, pp. 45-52, 2009.

[31] N. I. Mahmudov and S. Zorlu, "Controllability of semilinear stochastic systems," International Journal of Control, vol. 78, no. 13, pp. 997-1004, 2005.

[32] R. Sakthivel, S. Suganya, and S. M. Anthoni, "Approximate controllability of fractional stochastic evolution equations," Computers \& Mathematics with Applications, vol. 63, no. 3, pp. 660-668, 2012.

[33] R. Sakthivel, R. Ganesh, and S. Suganya, "Approximate controllability of fractional neutral stochastic system with infinite delay," Reports on Mathematical Physics, vol. 70, no. 3, pp. 291311, 2012.

[34] R. Sakthivel, J. J. Nieto, and N. I. Mahmudov, "Approximate controllability of nonlinear deterministic and stochastic systems with unbounded delay," Taiwanese Journal of Mathematics, vol. 14, no. 5, pp. 1777-1797, 2010.

[35] R. Sakthivel, Y. Ren, and N. I. Mahmudov, "Approximate controllability of second-order stochastic differential equations with impulsive effects," Modern Physics Letters B, vol. 24, no. 14, pp. 1559-1572, 2010.

[36] R. Sakthivel and E. R. Anandhi, "Approximate controllability of impulsive differential equations with state-dependent delay," International Journal of Control, vol. 83, no. 2, pp. 387-393, 2010.

[37] X. J. Li and J. M. Yong, Optimal Control Theory for InfiniteDimensional Systems, Birkhäuser, Boston, Mass, USA, 1995.

[38] V. Barbu and Th. Precupanu, Convexity and Optimization in Banach Spaces, vol. 10 of Mathematics and Its Applications (East European Series), D. Reidel Publishing, Dordrecht, The Netherlands, 2nd edition, 1986. 


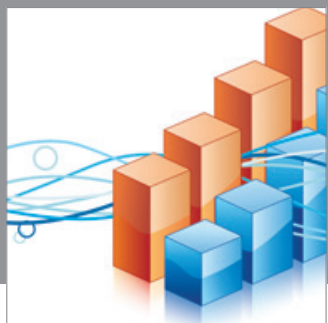

Advances in

Operations Research

mansans

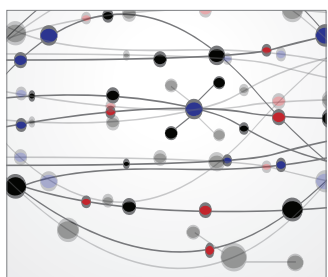

The Scientific World Journal
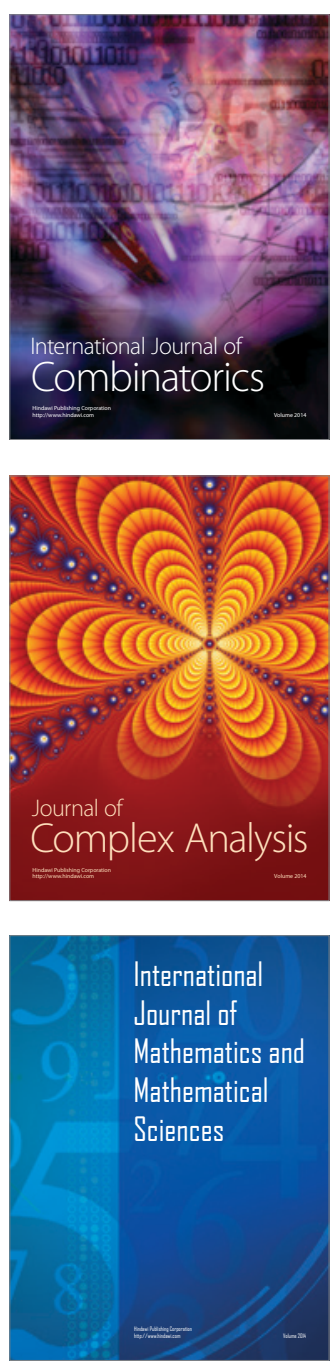
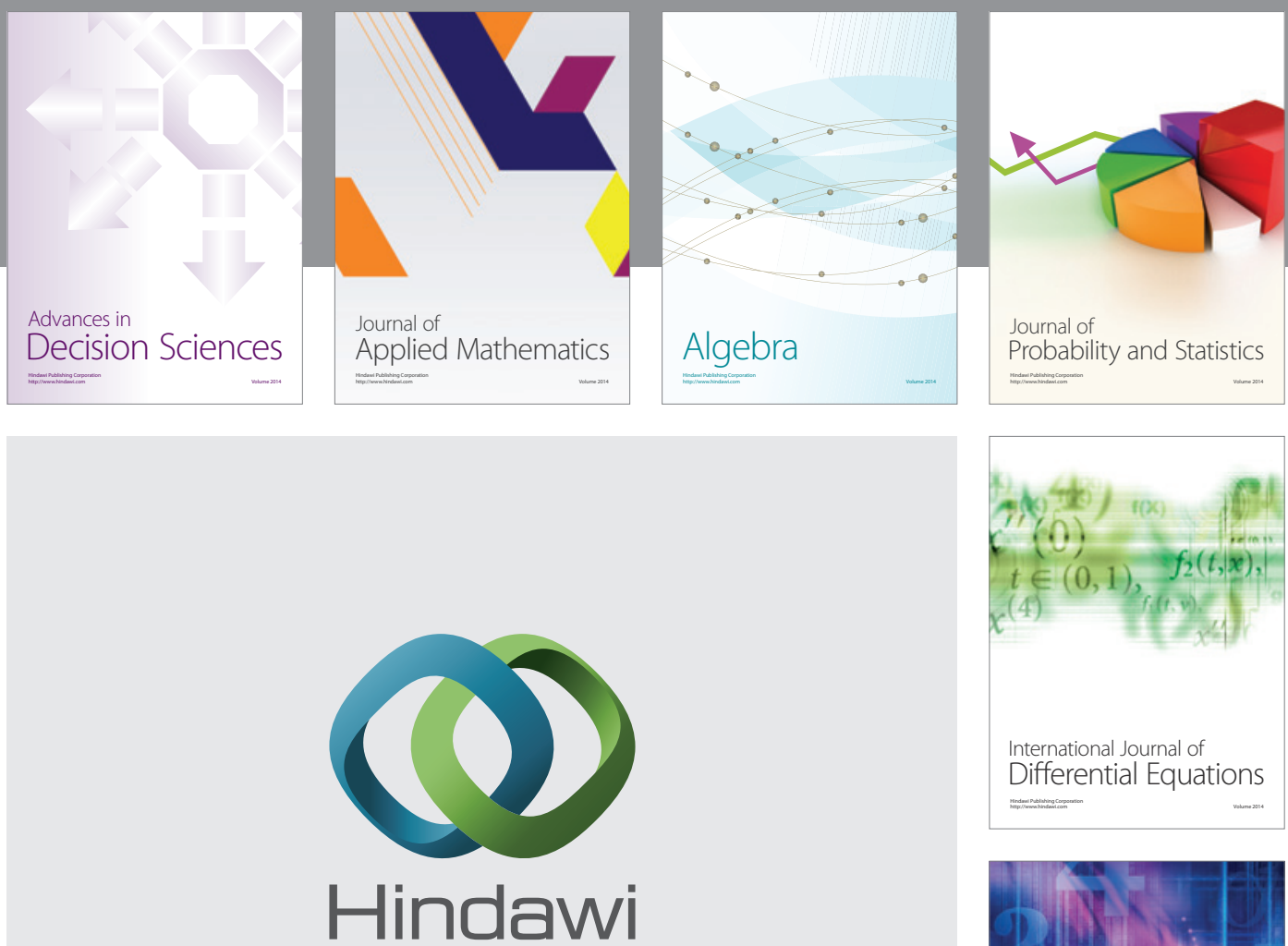

Submit your manuscripts at http://www.hindawi.com
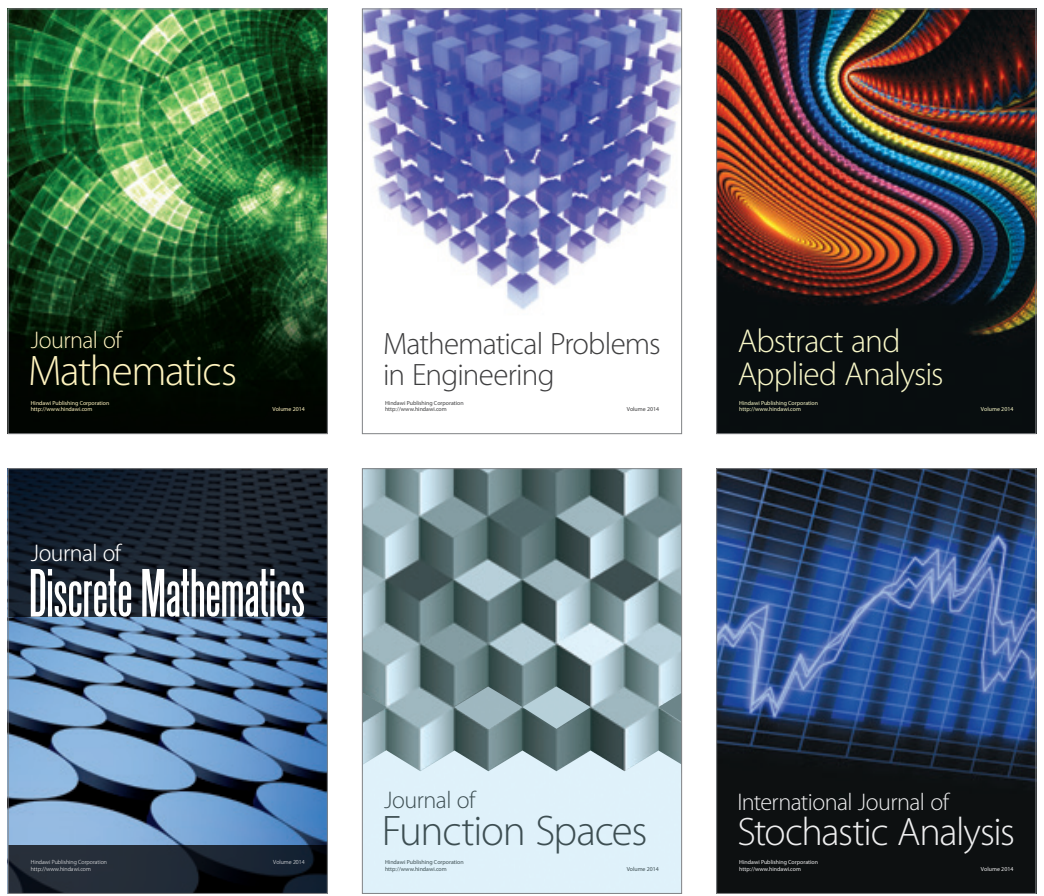

Journal of

Function Spaces

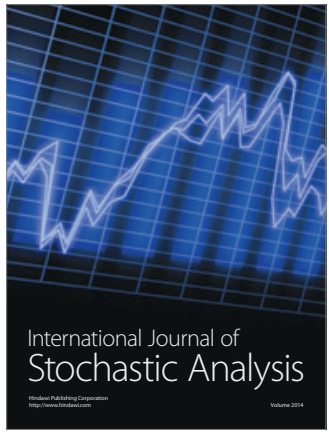

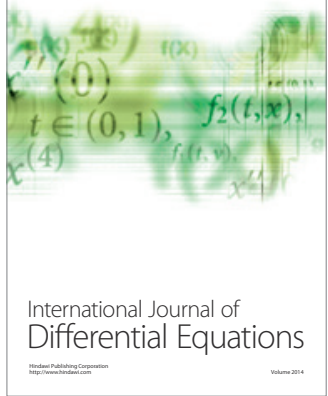
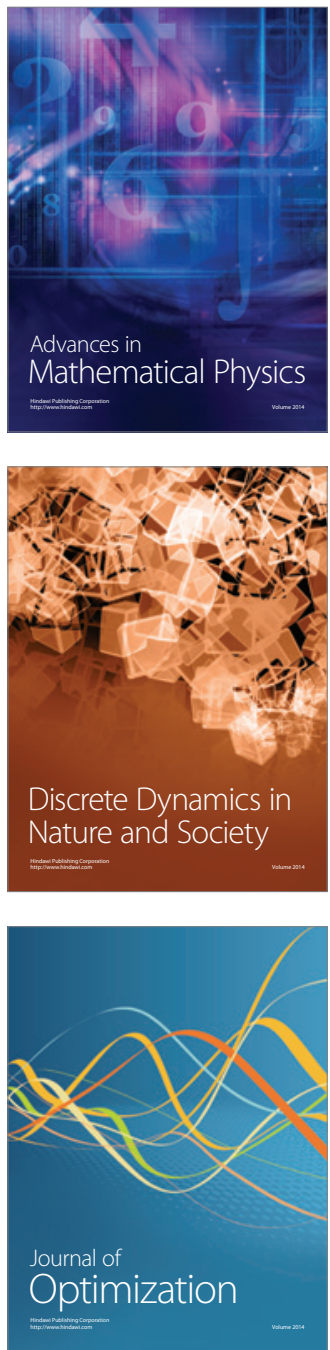